\title{
Modernización de los seruicios públicos municipales a través de las TIC, desde una perspectiva integral
}

\author{
Modernization of municipality public services through ICT, \\ from an integral perspective ${ }^{1}$
}

\author{
Patiño Galván Israel ${ }^{2}$ \\ Ceja Pizano J. Jesús ${ }^{3}$
}

\section{FORMA DE CITACIÓN}

Patiño, I, y Ceja, J. J. (2013), Modernización de los servicios públicos municipales a través de las tic, desde una perspectiva integral. Revista Dimensión Empresarial, vol. 11, núm. 2, pp. 70-88.

\section{RESUMEN}

Se propone modernizar los servicios públicos municipales, auxiliándose de las tecnologías de la información y comunicación, con la finalidad de mejorar la eficiencia y eficacia en la atención de los mismos, empleando metodologías científicas cualitativas, cuantitativas y metodologías tecnológicas de intervención que auxilien la correcta estructuración y fundamento de estos servicios. El resultado de esta investigación es la implementación de un sistema de información integrando componentes organizacionales, estratégicos y educativos para mejorar los procesos administrativos y operativos en la atención y seguimiento de dichos servicios. Para ello se recopiló la información de 6 municipios de la República Mexicana mediante técnicas de investigación de campo como son la entrevista y la observación, aplicadas al personal administrativo y operativo que presta dicho servicio en cada uno de los municipios, además de complementar éstas con la aplicación de 196 encuestas a los ciudadanos.

Palabras Clave: Control de Gestión, educación, tecnología, estrategias, Servicios Públicos Municipales.

\section{ABSTRAC}

It is proposed to modernize municipality public services, with the help of information technologies and communication, in order to improve efficiency and effectiveness in employing them, using scientific methodologies qualitative, quantitative and technological intervention methodologies that manage proper structuring and foundation of these services. A result of this research is the implementation of an information system integrating organizational, strategic and educational components to improve administrative and operational processes for the attention and follow-up of these services. This information was collected from six municipalities in Mexico through field research techniques such as interviews and observation, applied to administrative and operational staff that provides this service in each one of the municipalities, while complementing them with application of 196 surveys to citizens.

Keywords: Management Control, education, technology, strategies, municipality public services.

\section{INTRODUCCIÓN}

La evolución en la modernización de servicios públicos municipales en México se ha desarrollado en un ambiente en el que la demanda de éstos ha ido en aumento, derivado del incremento en la población. Según datos del INEGI en el 2005, México contaba con una población de 103.263.388 y pasó al 2010 a una población de 112.336.538, lo que representa un aumento del 8.7 \% en relación al 2005 .

A este incremento en la demanda de servicios públicos municipales, se suma el aspecto tecnológico de los sistemas de información, los cuales en las últimas décadas han tenido gran auge en el mundo y en México, ya que la

Artículo de investigación. Recibido en julio 11 de 2013. Aceptado en septiembre 23 de 2013.

Patiño Galván Israel, estudiante de doctorado del Instituto Politécnico Nacional de la Escuela Superior de Comercio y Administración, Unidad Santo Tomas, México, México D. F. Correo: ispa ga@hotmail.com

3 Ceja Pizano J. Jesús, profesor investigador del Instituto Politécnico Nacional y de la Universidad Nacional Autónoma de México, México D. F. Correo: cejapiza@yahoo.com 
incorporación de las mismas presiona su implementación en la administración pública municipal. Sin embargo, esta implementación y el uso de los sistemas de información deben de realizarse, con un adecuado análisis y planeación, no solo a corto plazo, sino con miras a un mediano y largo plazo. Por otro lado, las perspectivas en la modernización son alentadoras, ya que la implementación y el uso de las tecnologías de información en particular los sistemas de información, bien contextualizados social y normativamente, pueden ser una alternativa viable y objetiva para poder hacer frente a las demandas de servicios públicos.

Es importante mencionar que la educación no escapa a la evolución y perspectivas en la modernización de los servicios públicos municipales, ya que por un lado el aprovechamiento de los sistemas de información necesita de personal bien capacitado para realizar el análisis contextual de las necesidades y por otro, que los ciudadanos tengan el mínimo conocimiento tecnológico para que de igual manera aprovechen dichas tecnologías.

Así mismo, es necesario instrumentar mecanismos y programas que eleven los niveles académicos de la población (ciudadanos y autoridades) y también sobre la capacitación tecnológica continua. Por último, no se debe de dejar a un lado la implementación de estrategias para evaluar, medir y proponer alternativas de mejoras en el funcionamiento operativo y administrativo de todo el procedimiento que se lleva a cabo para la atención y seguimiento de los servicios públicos municipales.

En el caso de México, los gobiernos municipales han empezado a apoyarse en los sistemas de información para ofrecer un medio alterno para la solicitud y seguimiento de los servicios públicos municipales, y para valorar el proceso de modernización. Algunos municipios de México que han hecho este tipo de incorporaciones tecnológicas han obteniendo resultados favorables.

\section{CONSIDERACIONES METODOLÓGICAS}

\section{Tipo de investigación científica}

Por las características de esta investigación se considera que abarca los siguientes tipos de investigación: descriptiva, explicativa, de campo y propositiva.

La investigación descriptiva consiste en la caracterización de un hecho, fenómeno, individuo o grupo, con el fin de establecer su estructura o comportamiento. La investigación explicativa, se encarga de buscar el porqué de los hechos mediante el establecimiento de relaciones causa-efecto (Fernández, Narez \& García, 2008, 54-58). También se estará incluyendo la Investigación de campo, en la cual las principales técnicas que se emplearon fueron: la entrevista, la encuesta y la observación.

La encuesta es una técnica para adquirir información de interés sociológico, mediante un cuestionario previamente elaborado (Hernández, Collado \& Baptista, 1997, 28). Mientras que la entrevista estructurada según Sabino (2002) se caracteriza por estar rígidamente estandarizada, se plantean idénticas preguntas y en el mismo orden a cada uno de los participantes, quienes deben escoger la respuesta entre dos, tres o más alternativas que se les ofrecen.

Posteriormente la observación no participante la cual Hernández, Collado, \& Baptista (1997) indican que consiste en el registro sistemático, válido y confiable de comportamiento o conducta manifiesta. Por último, la investigación propositiva, la cual parte de un diagnóstico, se establecen metas y se diseñan estrategias para alcanzarlas (Del Rincon, Arnal, Latorre \& Sans, 1995, 25).

\section{Tipo de investigación tecnológica}

El tipo de investigación tecnológica empleada es de intervención, la cual según García (2005) es un elemento terminado, que reúne la aplicación del conocimiento en un producto específico que resuelve un problema, cubre una necesidad, facilita el quehacer, hace eficiente una tarea o brinda confort.

\section{Método de investigación científica}

Esta investigación se desarrolló desde el punto de vista cualitativo y cuantitativo, la finalidad de la investigación cualitativa, es comprender e interpretar la realidad, tal y como es entendida por los sujetos participantes en los contextos estudiados (Rodriguez, Gil, \& García, 1996, 1).

La investigación cuantitativa está directamente basada en el paradigma explicativo, en el cual se utiliza preferentemente información cuantitativa o cuantificable para describir o tratar de explicar los fenómenos que estudia (Briones, 2002, 17-18). Debido a la naturaleza de la presente investigación también se emplearon las siguientes metodologías:

1. Análisis y síntesis. El análisis es un procedimiento teórico mediante el cual un todo complejo se descompone en sus diversas partes y cualidades. Mientras que la síntesis establece mentalmente la unión entre las partes previamente analizadas y posibilita descubrir las relaciones esenciales y características generales entre ellas.

2. Sistémico estructural funcional, su acción se evidencia en la interrelación de las ideas, la conexión de los conceptos, los sistemas de recomendaciones y unido a las estrategias, se convierte en una vía importante para 
la explicación del objeto de investigación (Fernández, Narez \& García, 2008, 55-56).

3. Inductivo, Es el razonamiento, que partiendo del conocimiento de los caracteres necesarios de parte de los objetos de una clase, se infiere una conclusión universal acerca de los objetos de esa clase (Castillo de la Peña, 2010, 228-229).

\section{Método de investigación Tecnológica}

La metodología tecnológica es un modo sistemático de realizar, gestionar y administrar un proyecto para llevarlo a cabo con altas posibilidades de éxito. De lo anterior la metodología que se utilizó es la del ciclo de vida orientado a objetos, la cual, arma módulos basados en componentes, esto permite que el código sea reutilizable (Dante, 2006, 20-34). Dicha propuesta se auxiliará de los modelos administrativos existentes como el control de gestión municipal del Instituto Nacional de Administración Pública - INAP (1986), procedimientos administrativos actuales de los municipios, además de hacer uso de herramientas para dicho control como el Balance Scord Card de (Kaplan \& Norton, 2002). Además de retomar las experiencias de los sistemas ya creados como el desarrollado en el Municipio de Querétaro y la Delegación Miguel Hidalgo, y de los avances que en este tema han tenido los demás municipios investigados.

\section{CONTEXTODELOSSERVICIOS PÚBLICOS MUNICIPALES EN MÉXICO}

En México, se hace referencia a los Servicios Públicos, en la Constitución centralista de 1836, la primera en regular los ayuntamientos, al disponer, en la sexta de sus leyes, la existencia no de municipios sino de órganos colegiados electos popularmente en las capitales de los departamentos. Fue hasta 1917 en el artículo 115, Fracción III, de la Constitución Política de los Estados Unidos Mexicanos, donde se hace mención de los servicios públicos municipales, el cual dicta de la siguiente manera: Los Municipios con el concurso de los estados, cuando así fuere necesario y lo establezcan las leyes locales, tendrán a su cargo los siguientes servicios públicos:

1. Agua potable, alcantarillado, saneamiento y aguas residuales

2. Alumbrado público

3. Limpia y disposición de desechos

4. Mercados y centrales de abasto

5. Panteones

6. Rastro

7. Calles, parques, jardines, áreas verdes y recreativas

8. Seguridad pública y tránsito

9. Embellecimiento y conservación de los poblados, centros urbanos y obras de interés social

10. Asistencia social en el ámbito de su competencia,

11. De empleo.
Los demás servicios públicos que las legislaturas locales determinen, de acuerdo a las condiciones territoriales y socioeconómicas de los municipios, así como su capacidad administrativa y financiera.

En este contexto, cada estado y municipio tiene la normatividad respectiva, para la operación de dichos servicios públicos, tal y como lo indica la Constitución. Por citar un ejemplo de alguna normatividad que deben de seguir los municipios, como es el caso del municipio de Ecatepec de Morelos, Estado de México, se rige por la Constitución Política de los Estados Unidos Mexicanos, por la Constitución Política del Estado Libre y Soberano del Estado de México, por la Ley Orgánica Municipal, el Reglamento Interno Municipal y el Bando Municipal (Gobierno municipal de Ecatepec de Morelos, 2011).

\section{Clasificación de los servicios públicos municipales}

Según Serra (2002), los servicios públicos pueden clasificarse en cuatro categorías:

1. Servicios públicos federales.

2. Servicios públicos de las entidades federativas.

3. Servicios públicos municipales.

4. Servicios públicos internacionales.

\section{CONTEXTO INTERNACIONAL DE LAS TIC EN LA ADMINISTRACIÓN PÚBLICA}

Comenta Weissbluth (2008) que las TIC juegan un rol fundamental: son herramientas que están produciendo cambios radicales en la gestión pública, y paradójicamente, son las que más escapan a la comprensión de los reformadores que están gestionando o financiando proyectos de informatización pública. En este contexto indican Gaston \& Naser (2012) que debe considerarse que medir desempeño y avance de las políticas de gobierno electrónico trae consigo no sólo aspectos técnicos, sino que es necesario también considerar aspectos culturales, organizacionales, políticos, sociales. Por otra parte, comenta Penso (2011) que en el caso de México, éste enfrenta problemas para la utilización de las TIC como son: la preparación básica de los empleados para operar la tecnología, el alto grado de subutilización de la capacidad instalada, pero sobre todo la presión que recae sobre el empleado para que se autocapacite.

\section{Modelos de evolución del gobierno electrónico}

Comentan Gaston \& Naser (2012) que la Organización de las Naciones Unidas ha definido un marco de evolución del gobierno electrónico según cuatro niveles de madurez, el cual ha servido de referencia para que los países definan sus propios modelos: 
a) Nivel emergente: El país asume el compromiso de desarrollar el gobierno electrónico, ofreciendo información en una sola vía (del gobierno a la ciudadanía).

b) Nivel mejorado: Se amplía la comunicación de una a dos vías, con la interacción de la ciudadanía mediante correo electrónico, formularios descargables, aplicaciones, audio, video y la presentación de solicitudes.

c) Nivel transaccional: El Estado ofrece transacciones completas y seguras como el pago de impuestos y multas, inscripciones, obtención de permisos, certificados y licencias, entre otros.

d) Nivel conectado: Información, datos y conocimiento se transfieren entre organismos, para un acceso instantáneo a servicios del Estado de manera integrada y con un enfoque centrado en el ciudadano.

\section{Proceso de incorporación de las TIC}

El proceso de incorporación de las TIC, según Montaño (2008) es el siguiente:

1. Preparación del entorno.

2. Sensibilización de la Innovación.

3. Exploración de Recursos Tecnológicos.

4. Exploración de Recursos Educativos.

5. Generación de propuestas o prototipos.

6. Retroalimentación.

\section{Beneficio del gobierno electrónico}

Una forma útil de presentar estos beneficios es entendiéndolos como resultados de ciertas acciones e iniciativas de gobierno electrónico. En este sentido, los aportes podrían resumirse de la siguiente manera: mejoramiento de la calidad de los servicios públicos, eficiencia y productividad en los procesos y operaciones gubernamentales, transparencia y rendición de cuentas, participación ciudadana, marco regulatorio que respalde el gobierno electrónico.

\section{Tendencias del gobierno electrónico}

Se presentan a continuación según Gaston \& Naser (2012), las tendencias sobre los aspectos que actualmente se están desarrollando con más fuerza en países desarrollados y que probablemente tocará afrontar en la región de América Latina en un plazo breve, con la ventaja de incorporar las lecciones y adaptaciones, siempre que se esté efectivamente preparado para aprender de éstas.

- Recuperación de desastres

- Cloud computing

- Participación ciudadana

- Indicadores de gobierno electrónico

- Gobierno abierto

\section{CONTEXTO DE LOS SISTEMAS DE INFORMACIÓN}

Los sistemas de información, señalan Andreu \& Valor (1991) son un conjunto formal de procesos que operan sobre una colección de datos estructurada de acuerdo a las necesidades de la empresa. Recopilan, elaboran y distribuyen selectivamente la información necesaria para la operación de dicha empresa.

\section{Clasificación de los sistemas de información}

Según Edwars, Ward \& Bythesway (1998) y García (2000) los sistemas se clasifican de la siguiente manera:

1. Grado de formalidad

2. Automatización

3. Relación con la toma de decisiones

4. Funcionalidad

5. Grado Especialización

Laudon \& Laundon (1996), establecen la siguiente clasificación de los sistemas de información:

1. Sistema de Procesamiento de Operaciones

2. Sistemas de Trabajo del Conocimiento

3. Sistemas de Automatización en la Oficina

4. Sistemas de Información para la Administración

5. Sistemas para el Soporte de Decisiones

6. Sistemas de Soporte Gerencial

\section{MODERNIZACIÓN DE LOS SERVICIOS PÚBLICOS MUNICIPALES EN MUNICIPIOS DE MÉXICO: TRES EXPERIENCIAS PARA EL ANÁLISIS}

La modernización de los servicios públicos municipales, no ha sido similar en los municipios de México, en algunos municipios como Querétaro, Ecatepec y la delegación Miguel Hidalgo de la ciudad de México, ya que se están apoyando del uso de tecnologías de la información / sistemas de información. Sin embargo, el hecho de auxiliarse de Sistemas de Información para para su modernización, no significa eliminar los procesos tradicionales, sino más bien establecer un programa para que paulatinamente se realice dicha incorporación.

A continuación se muestran en la Gráfica 1, los habitantes por $\mathrm{Km}^{2}$, de algunos municipios para dimensionar dicha cobertura.

En la Gráfica 2, se muestran los datos sobre el número de solicitudes de servicios públicos municipales y el incremento promedio de solicitudes vía tecnologías de información / Sistemas de Información, y los niveles de educación y alcance en las tecnologías. 
Gráfica 1. Habitantes por kilómetro cuadrado, de los municipios de Ecatepec, Coacalco, Nezahualcóyotl y Tecámac, Estado de México, y los municipios de Querétaro, Pachuca de Soto y la Delegación Miguel Hidalgo

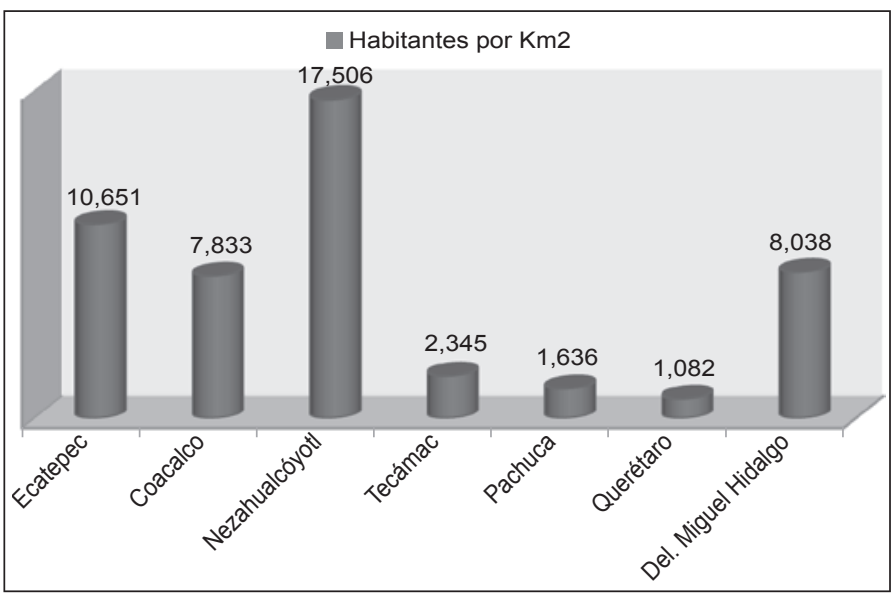

Fuente elaboración propia (2012). Con base a los datos del INEGI 2010, http://www.inegi.org.mx/sistemas/mexicocifras, recuperado el 01/02/2011. Los habitantes por $\mathrm{Km}^{2}$, fue elaboración propia dividiendo la población entre la superficie $/ \mathrm{Km}^{2}$.

Gráfica 2. Comportamiento de los indicadores de servicios públicos, respecto a la educación y alcance en las tecnologías de la información

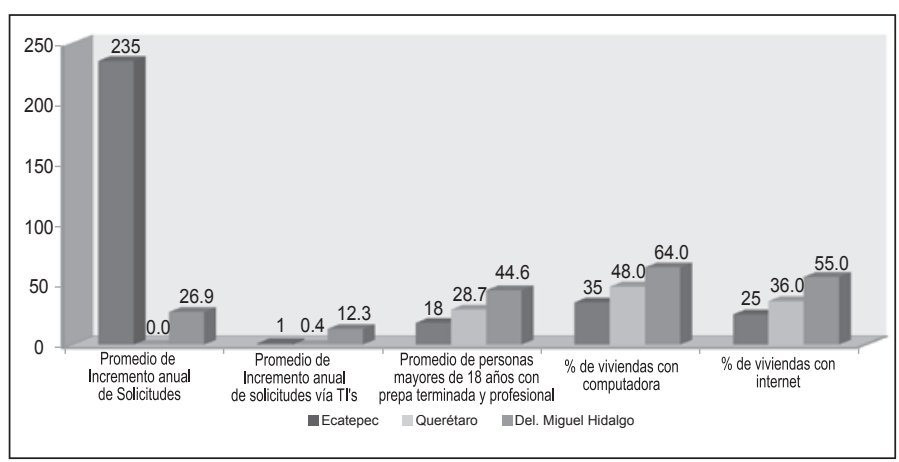

Fuente: Elaboración propia (2012), con base a los siguientes datos: Ecatepec. Información proporcionada por la unidad de transparencia del Municipio de Ecatepec, vía electrónica, mediante el SICOSIEM (Sistema de Control de Solicitudes de Información del Estado de México), folio asignando a la solicitud 00656 y 00654, fecha de solicitud de información 28/06/2011. Querétaro. Información proporcionada por el centro de servicios y atención ciudadana, vía electrónica, mediante el sistema de solicitudes de información de Querétaro (SSIQ), folio asignando a la solicitud 716 y 717 , Fecha de solicitud de información 11/08/2011. Miguel Hidalgo. Información proporcionada por el centro de servicios y atención ciudadana, vía electrónica, mediante el sistema INFOMEX (sistema de solicitudes de información del distrito federal), folio asignando a la solicitud 3 y 5 , fecha de solicitud de información 26/06/2011. INEGI.2010. http://www3.inegi.org.mx/sistemas/ TabuladosBasicos/Default.aspx?c=27302\&s=est, recuperado el 25/07/2011.

El comportamiento en dichas gráficas indica que el aumento en la utilización de tecnologías de información / sistemas de información, está ligado a la educación y alcance de la tecnología, con lo que se incrementan paulatinamente las solicitudes de un servicio público mediante un sistema de información. Aunado a la difusión municipal en la utilización de ese medio tecnológico para la solicitud de dichos servicios. La utilización de la tecnología, da una perspectiva alentadora, ya que, el control de gestión de un servicio público municipal mediante los sistemas de información ha tenido aceptación y resultados en los casos de los municipios de Querétaro y la Delegación Miguel Hidalgo de la ciudad de México, donde ya utilizan este medio tecnológico para el control y seguimiento de servicios públicos municipales.

En el caso del municipio de Querétaro, Estado de Querétaro, se cuenta con un sistema de información para el control de Gestión de los servicios públicos municipales. En éste, independientemente de cual sea el ingreso de la solicitud: en persona, por documento, teléfono, etc., todos son ingresadas al sistema de información, con lo cual se garantiza un solo canal de comunicación, ya que la solicitud es hecha por el ciudadano, y éste es atendido por dicha área de servicios públicos, no se tiene que andar buscando el área que tiene su solicitud. Una vez que ingresa la solicitud al sistema, la comunicación con las áreas administrativas y operativas que brindan el servicio, se lleva a cabo mediante dicho sistema, como a continuación se muestra en la llustración 1.

Por otro lado, en municipios que recientemente están en el proceso de incorporar tecnologías de información, tal es el caso del municipio de Ecatepec, Estado de México. Se tienen tres opciones para la solicitud de un servicio público municipal:

Opción 1. El ciudadano realiza la solicitud vía un documento a la Oficialía de Partes, la cual le asigna un folio y canaliza la solicitud al área de atención ciudadana de la dirección o secretaría que corresponda, dependiendo del servicio que se solicite. Esta opción es la más tardada ya que pasa por una instancia adicional que es Oficialía de Partes. Opción 2. El ciudadano realiza la solicitud directamente a las unidades de Atención Ciudadana (A.C) del servicio público municipal que requiera, una vez que Atención Ciudadana lo recibe, le entrega un número de folio para dar seguimiento a la solicitud, y esta unidad a su vez canaliza la solicitud a la dirección correspondiente para ser atendida. Finalmente, opción 3. El ciudadano realiza la solicitud vía correo electrónico / Facebook / Twitter, llegándole en ocasiones directamente al titular de cada dirección, o en su defecto al área de Atención Ciudadana. Posteriormente esta solicitud es atendida como si fuera una solicitud de la opción 2, la diferencia es que es atendida con mayor prontitud, ya que le llega directamente a la dirección sin pasar por el filtro de Oficialía de Partes y en ocasiones Atención Ciudadana, de igual manera se le asigna un número de folio para su seguimiento. A continuación se presenta la llustración 2, correspondiente a dicho municipio.

Se presenta a continuación la llustración 3, sobre el Control de Gestión de la delegación Miguel Hidalgo de la ciudad de México. 
Ilustración 1. Control de gestión, para la atención y seguimiento de los servicios públicos municipales, en el municipio de Querétaro, Estado de Querétaro

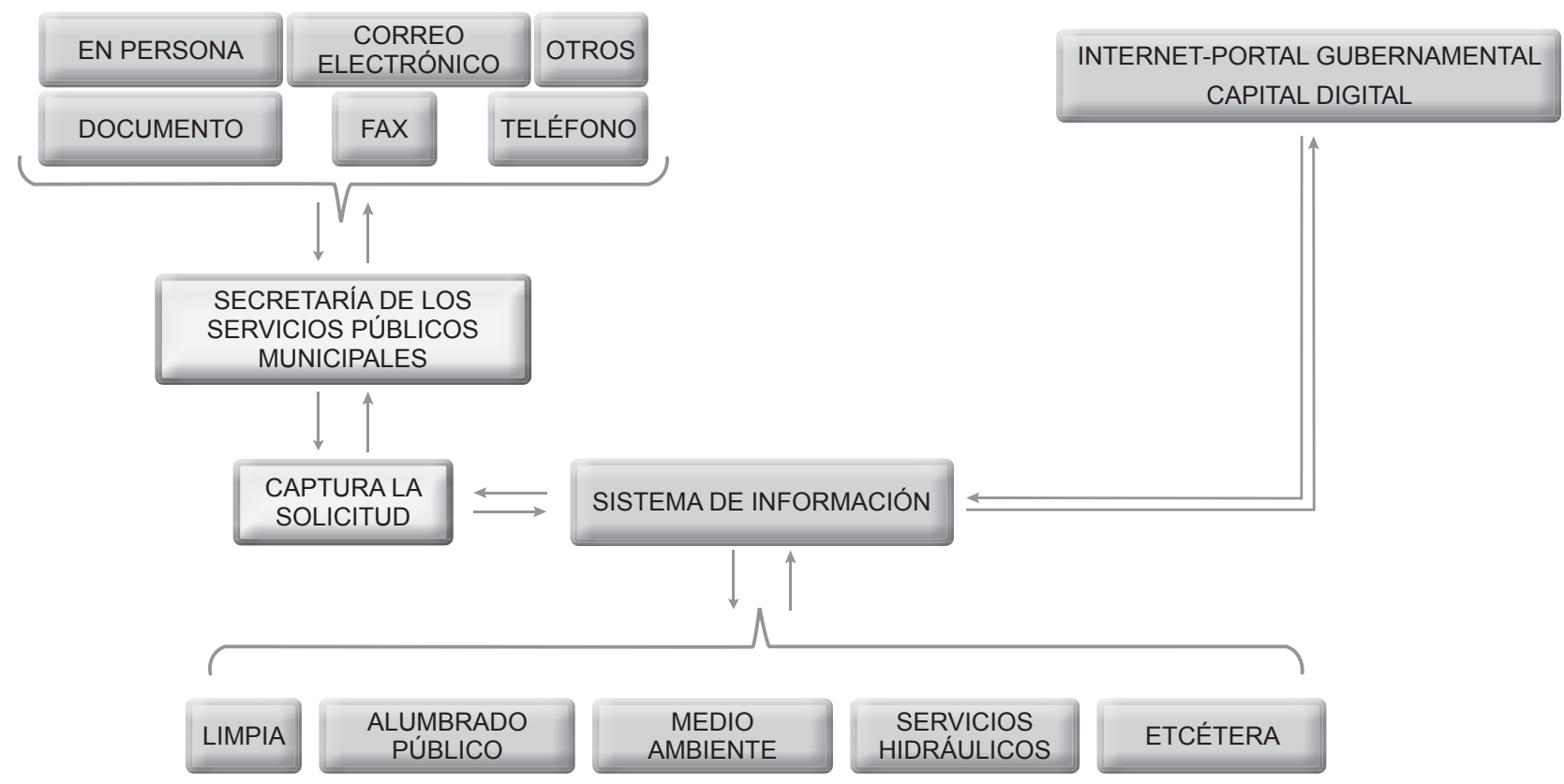

Fuente: Elaboración propia, derivada de la investigación de campo (Entrevista y observación) realizada el 22/11/2011. Exploración y análisis de la liga de internet del Gobierno Municipal de Querétaro, de las dependencias que ofrecen el Servicio Público Municipal del Municipio de Querétaro: http://www.municipiodequeretaro.gob.mx/, recuperado el 24/11/2011.

Ilustración 2. Diagrama de Control de Gestión, para la atención y seguimiento de los servicios públicos municipales de Ecatepec, Estado de México

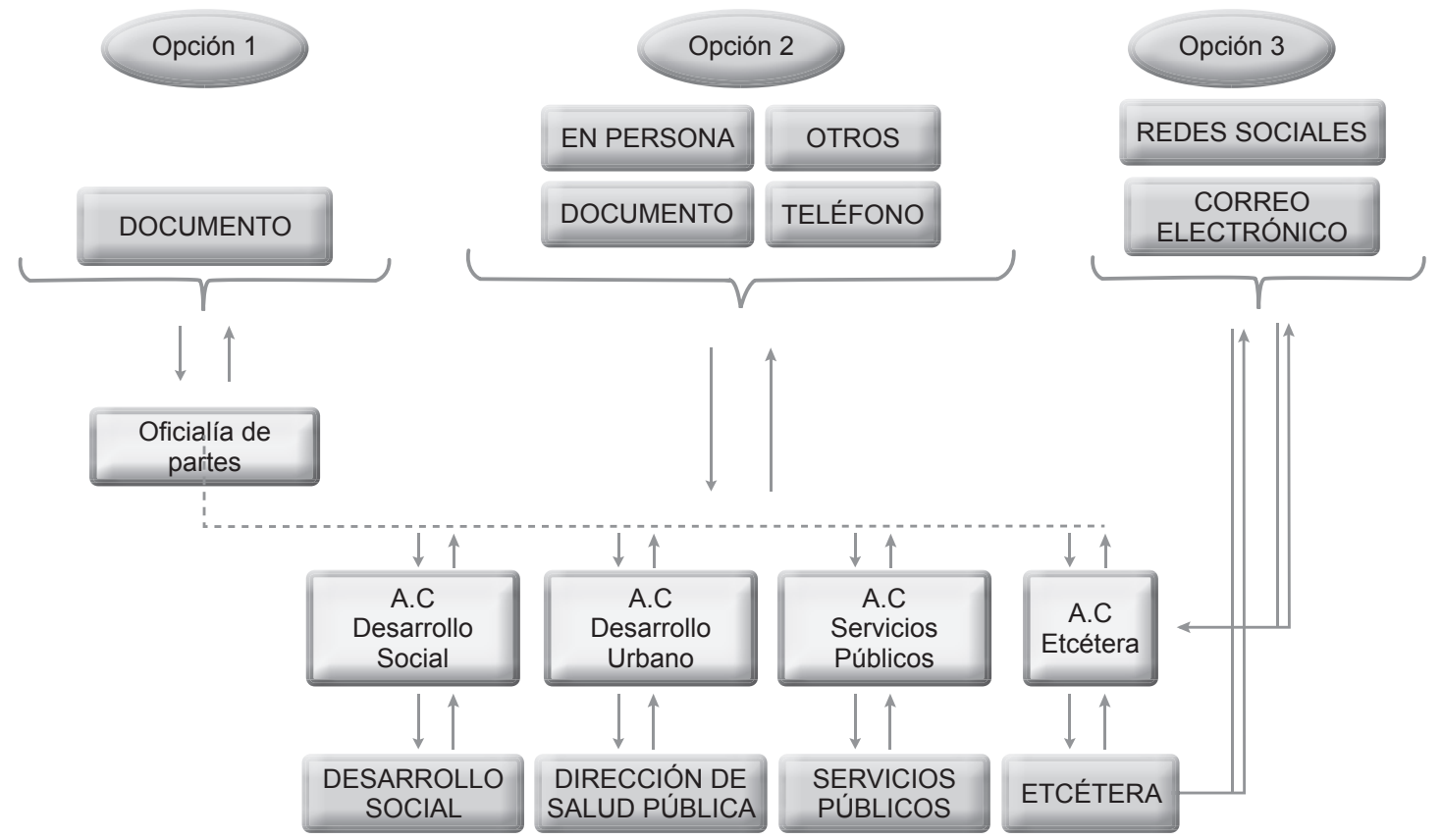

Fuente: Elaboración propia, derivada de la investigación de campo (entrevista y observación) realizada el 23/06/2011. Exploración y análisis de la liga de internet del Gobierno Municipal de Ecatepec, de las dependencias que ofrecen el Servicio Público Municipal: Ecatepec de Morelos: Ecatepec de Morelos: http://www.ecatepec.gob.mx/, Consultado el 23/06/2011 
Ilustración 3. Diagrama de control de gestión, para la atención y seguimiento de los servicios públicos municipales de la Delegación Miguel Hidalgo, de la ciudad de México

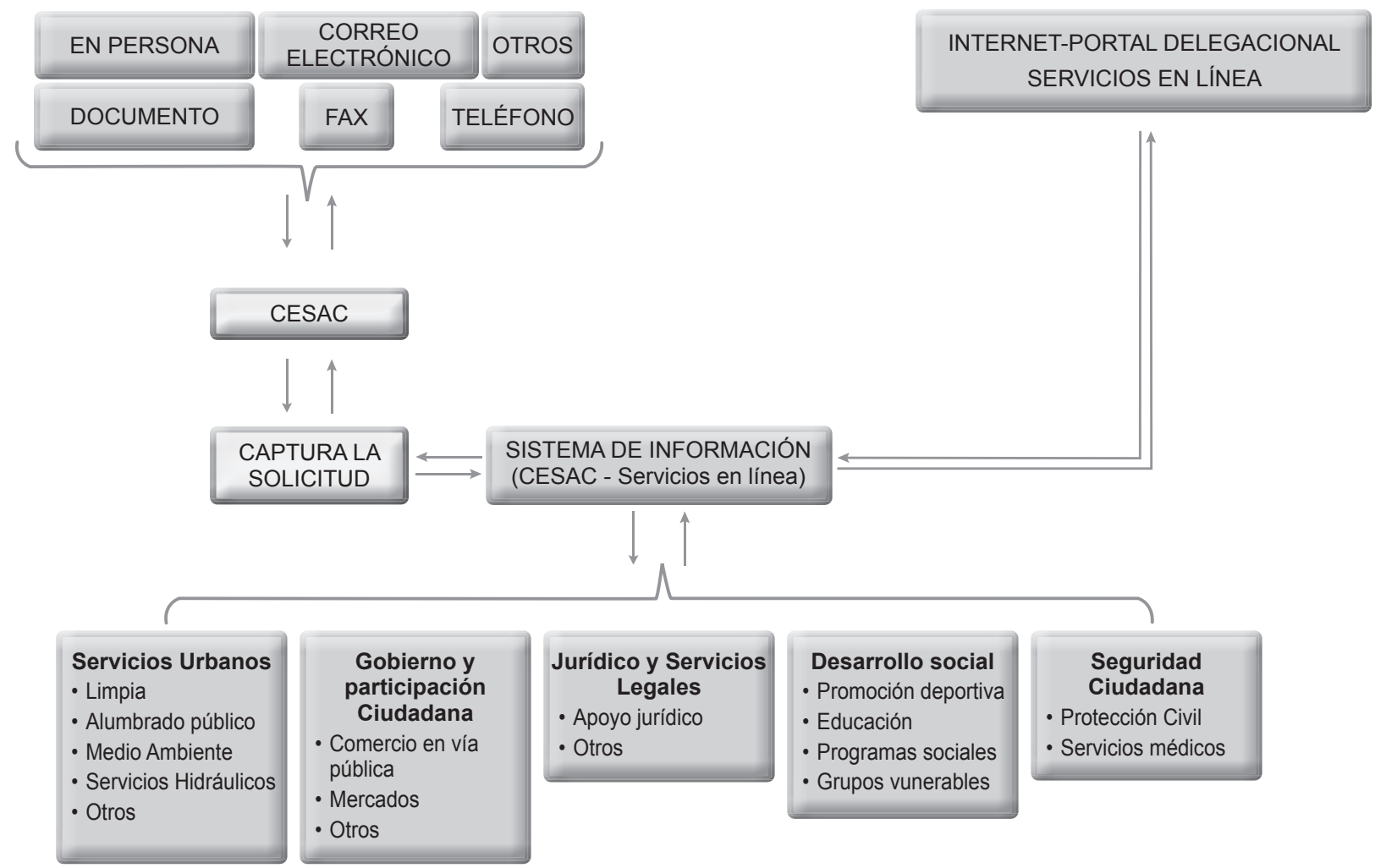

Fuente: Elaboración propia, derivada de la investigación de campo (entrevista y observación) realizada el 23/06/2011. Exploración y análisis de la liga de internet de la delegación Miguel Hidalgo, de las direcciones/dependencias que ofrecen el Servicio Público: Delegación Miguel Hidalgo: http://www.miguelhidalgo.gob.mx/, recuperado el 03/11/2011

\section{ESTRATEGIAS DE MODERNIZACIÓN DE LOS SERVICIOS PÚBLICOS MUNICIPALES A TRAVÉS DE LAS TIC, DESDE UNA PERSPECTIVA INTEGRAL}

\section{Componentes del modelo propuesto}

Si bien la propuesta principal es un sistema de información (componente tecnológico), se considera importante integrar a otros tres componentes para implementar ésta de manera satisfactoria. Se propone que los cuatro componentes se incorporen y operen de manera gradual, con el fin de plantear estrategias para que la población y autoridades se incorporen de manera paulatina, y a su vez los componentes propuestos logren su madurez, a excepción del componente educativo que requiere de un proceso de mayor madurez.

La implementación de la propuesta, está basada en 8 fases las cuales son: Planificación, Análisis, Diseño, Especificación, Implementación, Validación, Evaluación - Mantenimiento y Revisión.

1. El componente tecnológico. Consta de 4 fases

2. El componente organizacional. Se propone sea de 2 fases
3. El componente estratégico. Consta de 2 meses.

4. El componente educativo. Se estima que en los primeros 6 meses se puedan tener resultados favorables en este componente, por lo que se considera como una primera fase de seis meses de manera continua.

Se propone que dichas fases se incorporen de manera paralela, lo cual indica que en un lapso de 4 meses se estén integrando totalmente los primeros 3 componentes y el componente educativo se incorpore de manera continua cada seis meses. Se presenta a continuación en la llustración 4 el tiempo propuesto de integración de los cuatro componentes.

Se pueden observar las fases y el tiempo en las que se propone incorporar a los componentes, los tiempos de integración son de manera paralela. Cabe mencionar que la utilización de este modelo ya implementado, estará disponible para todos los ciudadanos de manera paulatina a partir del término de la segunda y tercera fase de los componentes tecnológico y organizacional (respectivamente). Se presenta a continuación en la Tabla 1, el cronograma conceptual de las 4 fases propuestas. 
Ilustración 4.Tiempo propuesto de integración total de los componentes

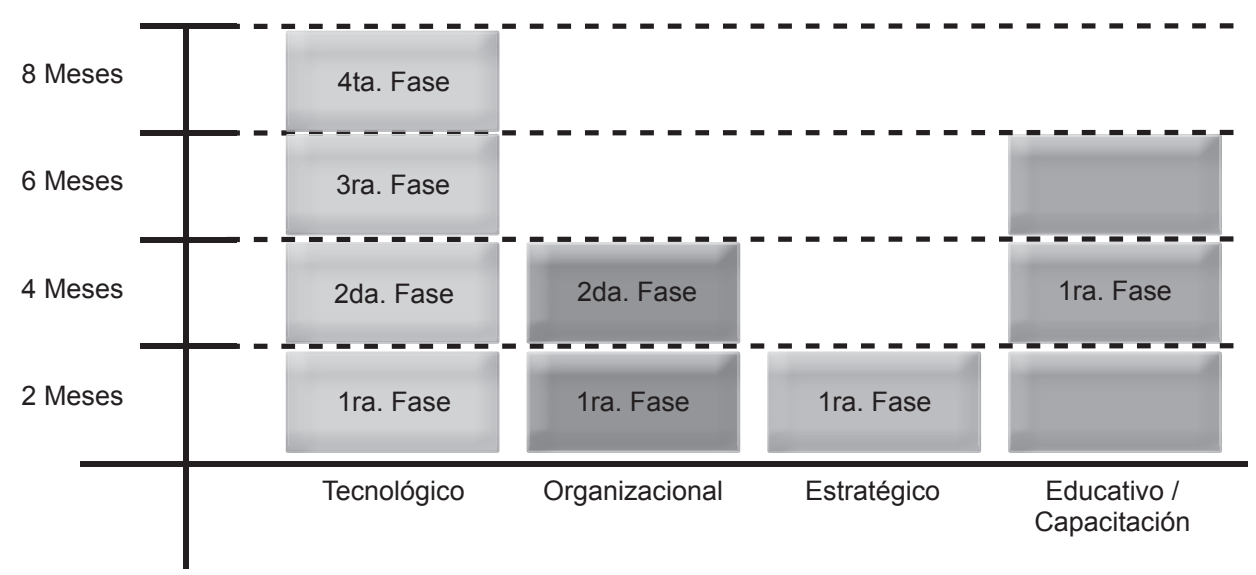

Fuente: Elaboración propia (2012)

Tabla 1. Cronograma propuesto de implementación de los cuatro componentes.

\begin{tabular}{|c|c|c|c|c|}
\hline Fases & Componente Tecnológico & $\begin{array}{l}\text { Componente } \\
\text { Organizacional }\end{array}$ & $\begin{array}{l}\text { Componente } \\
\text { estratégico }\end{array}$ & Componente educativo \\
\hline \multirow{4}{*}{$\begin{array}{l}\text { Primera } \\
\text { Fase }\end{array}$} & $\begin{array}{l}\text { Finalizar la funcionalidad del } \\
\text { sistema }\end{array}$ & $\begin{array}{l}\text { Definición de la estructura de } \\
\text { la Dirección propuesta }\end{array}$ & $\begin{array}{l}\text { Integración de los indi- } \\
\text { cadores al sistema de } \\
\text { información }\end{array}$ & $\begin{array}{l}\text { Se inicia planificación y es- } \\
\text { tructura de los programas } \\
\text { de capacitación tecnológica. }\end{array}$ \\
\hline & $\begin{array}{l}\text { Finalizar la integración de los } \\
\text { procedimientos administrativos y } \\
\text { operativos que se sistematizarán }\end{array}$ & $\begin{array}{l}\text { Integración del personal que } \\
\text { formará la estructura }\end{array}$ & $\begin{array}{l}\text { Definición de las fuen- } \\
\text { tes que alimentarán los } \\
\text { indicadores }\end{array}$ & $\begin{array}{l}\text { Se realiza la planificación } \\
\text { de fechas y lugares de ca- } \\
\text { pacitación. }\end{array}$ \\
\hline & $\begin{array}{l}\text { Liberación del sistema en am- } \\
\text { biente de desarrollo y pruebas }\end{array}$ & & \multirow{2}{*}{$\begin{array}{l}\text { Al término de la pre- } \\
\text { sente fase deberá de } \\
\text { incorporarse en su to- } \\
\text { talidad el componente } \\
\text { estratégico al sistema } \\
\text { de información }\end{array}$} & \multirow{2}{*}{$\begin{array}{l}\text { Se iniciará la gestión corres- } \\
\text { pondiente entre las autorida- } \\
\text { des para la planificación y } \\
\text { estrategia correspondiente } \\
\text { para iniciar con el compo- } \\
\text { nente educativo, en aras } \\
\text { de incrementar los niveles } \\
\text { educativos. }\end{array}$} \\
\hline & $\begin{array}{l}\text { Finalización de manuales de } \\
\text { usuario y técnicos del sistema }\end{array}$ & & & \\
\hline \multirow[b]{2}{*}{$\begin{array}{l}\text { Segunda } \\
\text { fase }\end{array}$} & $\begin{array}{l}\text { Integración del control de acceso } \\
\text { derivado de la integración del } \\
\text { personal que integrará la direc- } \\
\text { ción propuesta en el componente } \\
\text { organizacional }\end{array}$ & $\begin{array}{l}\text { Se podrá operar el sistema de } \\
\text { información, integrando los pro- } \\
\text { cedimientos administrativos y } \\
\text { operativos }\end{array}$ & \multirow{2}{*}{$\begin{array}{l}\text { Se podrá iniciar con } \\
\text { el funcionamiento y } \\
\text { análisis resultados del } \\
\text { componente estraté- } \\
\text { gico, para iniciar con } \\
\text { la operación de este } \\
\text { componente desde el } \\
\text { sistema de información }\end{array}$} & \multirow[t]{2}{*}{$\begin{array}{l}\text { Se inicia la capacitación tec- } \\
\text { nológica básica del personal } \\
\text { que operará el sistema, así } \\
\text { como la capacitación sobre } \\
\text { las tecnologías de informa- } \\
\text { ción }\end{array}$} \\
\hline & $\begin{array}{l}\text { Se realizan las fases de: Imple- } \\
\text { mentación del sistema de infor- } \\
\text { mación }\end{array}$ & $\begin{array}{l}\text { En esta fase deberá de haberse } \\
\text { terminado con la incorporación } \\
\text { del componente organizacional }\end{array}$ & & \\
\hline
\end{tabular}




\begin{tabular}{|c|c|c|c|}
\hline \multirow{3}{*}{$\begin{array}{l}\text { Tercera } \\
\text { fase }\end{array}$} & \multirow[t]{3}{*}{$\begin{array}{l}\text { Validación integral del siste- } \\
\text { ma de información., incluyendo } \\
\text { funcionalidad, procedimientos } \\
\text { e indicadores del componente } \\
\text { estratégico }\end{array}$} & $\begin{array}{l}\text { Se continua de manera perma- } \\
\text { nente con los programas de } \\
\text { cobertura, difusión y alcance, } \\
\text { del componente organizacional }\end{array}$ & $\begin{array}{l}\text { Se inicia la apertura de } \\
\text { cursos de capacitación a la } \\
\text { población, implementando } \\
\text { estrategias para lograr una } \\
\text { mejor cobertura, difusión y } \\
\text { alcance, con el objetivo de } \\
\text { hacer permanente dicha ca- } \\
\text { pacitación. }\end{array}$ \\
\hline & & \multirow{2}{*}{$\begin{array}{l}\text { Finalmente se estará en con- } \\
\text { diciones de ampliar el uso del } \\
\text { sistema a la población. Es im- } \\
\text { portante que esta apertura este } \\
\text { acompañada de previas capa- } \\
\text { citaciones y de la aplicación } \\
\text { de programas de cobertura, } \\
\text { difusión y alcance del compo- } \\
\text { nente organizacional }\end{array}$} & $\begin{array}{l}\text { Se recomienda continuar } \\
\text { con la aplicación de las es- } \\
\text { trategias del componente } \\
\text { educativo para lograr de ma- } \\
\text { nera paulatina el incremento } \\
\text { en los niveles educativos }\end{array}$ \\
\hline & & & $\begin{array}{l}\text { Al término de la fase } 3 \text {, de- } \\
\text { rivado de la integración del } \\
\text { componente educativo, se } \\
\text { espera tener resultados pau- } \\
\text { latinos, sobre el incremento } \\
\text { en los niveles educativos de } \\
\text { la población (autoridades y } \\
\text { ciudadanos) }\end{array}$ \\
\hline $\begin{array}{l}\text { Cuarta } \\
\text { fase }\end{array}$ & $\begin{array}{l}\text { Se realizará la evaluación-man- } \\
\text { tenimiento, así como la revisión } \\
\text { correspondiente de cada parte } \\
\text { que integra dicho componente } \\
\text { que se incluye dentro del com- } \\
\text { ponente tecnológico. }\end{array}$ & & \\
\hline
\end{tabular}

Fuente: Elaboración propia (2012).

La propuesta consta de cuatro componentes, que interactúan entre ellos para lograr una mejor eficiencia en el control de gestión de los servicios públicos municipales.

\section{Componente organizativo}

Su objetivo es proponer una estructura organizacional que maximice los recursos humanos y tecnológicos con que cuenta cada una de las unidades o dependencias que proporcionen algún servicio público municipal, respetando el marco normativo correspondiente, como se indica en Ilustración 5. Se propone crear una Dirección de Atención Ciudadana de los servicios públicos municipales que concentre a las unidades de atención ciudadana, y sea éste el único enlace con el ciudadano, con lo cual se garantiza una sola línea de comunicación con el ciudadano. La interacción con las áreas internas y externas se muestra en la llustración 6.

Ilustración 5. Estructura organizativa propuesta para la Dirección de Atención Ciudadana.

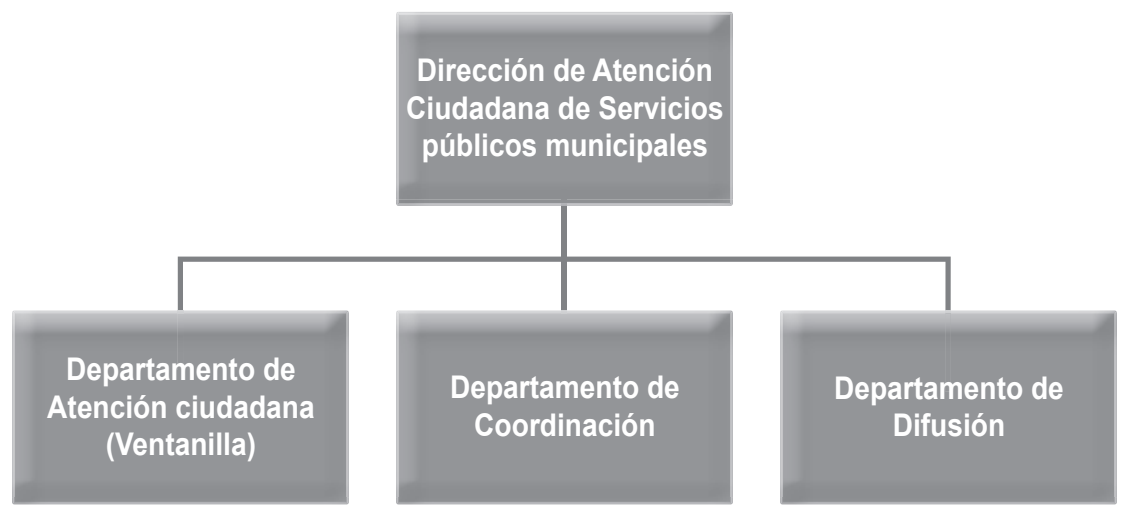

Fuente: elaboración propia (2012). 
Ilustración 6. Interacción de la Dirección de Atención Ciudadana

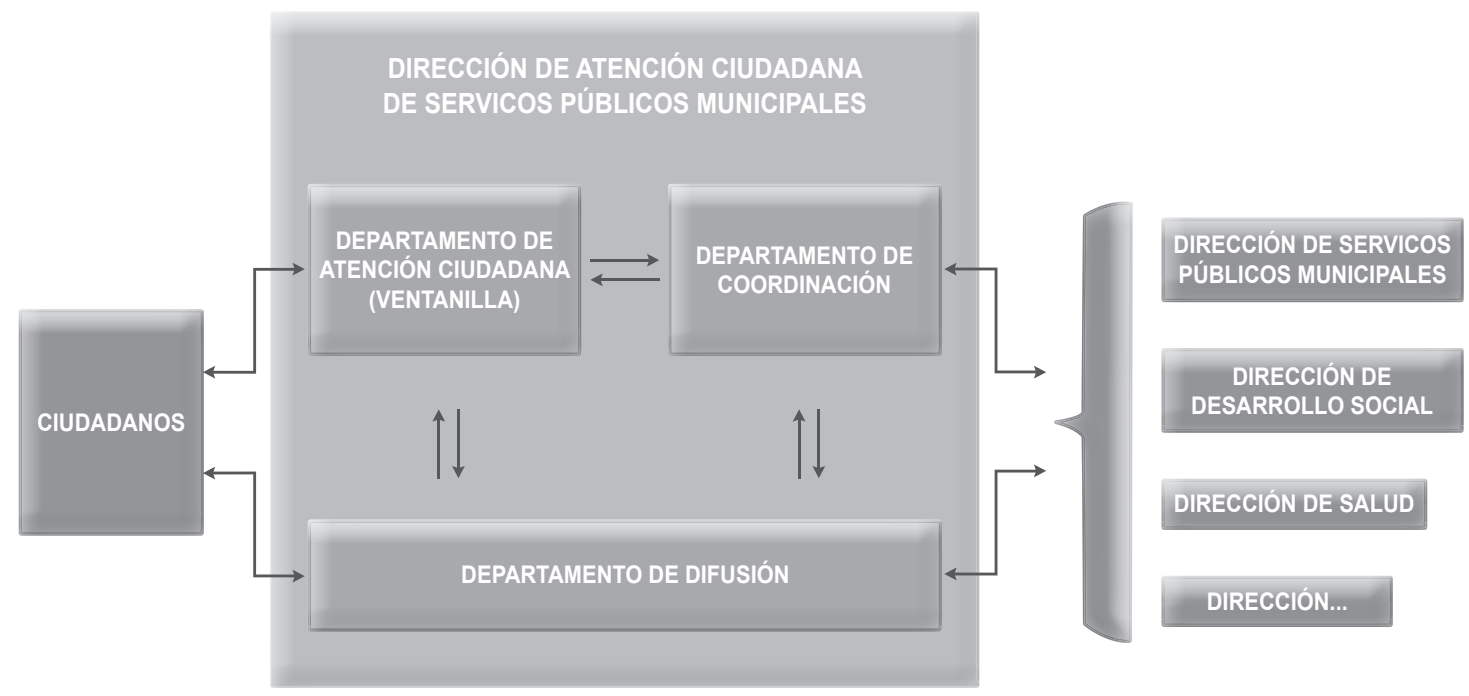

Fuente: elaboración propia (2012).

\section{Componente estratégico}

Proponer indicadores que permitan auditar el cumplimiento de los programas de trabajo, evaluar su realización, detectar desviaciones y proponer medidas correctivas, fortaleciendo con ello la coordinación y toma de decisiones de las direcciones y departamentos involucrados, y todo ello sirva para llevar a cabo un adecuado control de gestión de los servicios públicos municipales. Los indicadores propuestos se pueden observar en la llustración 7.

\section{Componente educativo}

Proponer alternativas para mejorar los niveles educativos de la población. Se busca capacitar tecnológicamente de manera continua a los ciudadanos, aprovechando la estructura pública con la que cuentan los municipios, propiciando con ello una satisfactoria implementación del componente tecnológico, fortaleciendo el aspecto educativo, reduciendo gradualmente el rezago que se tiene y que tanto adolecen los municipios de México.

Ilustración 7. Propuesta de indicadores del componente estratégico.

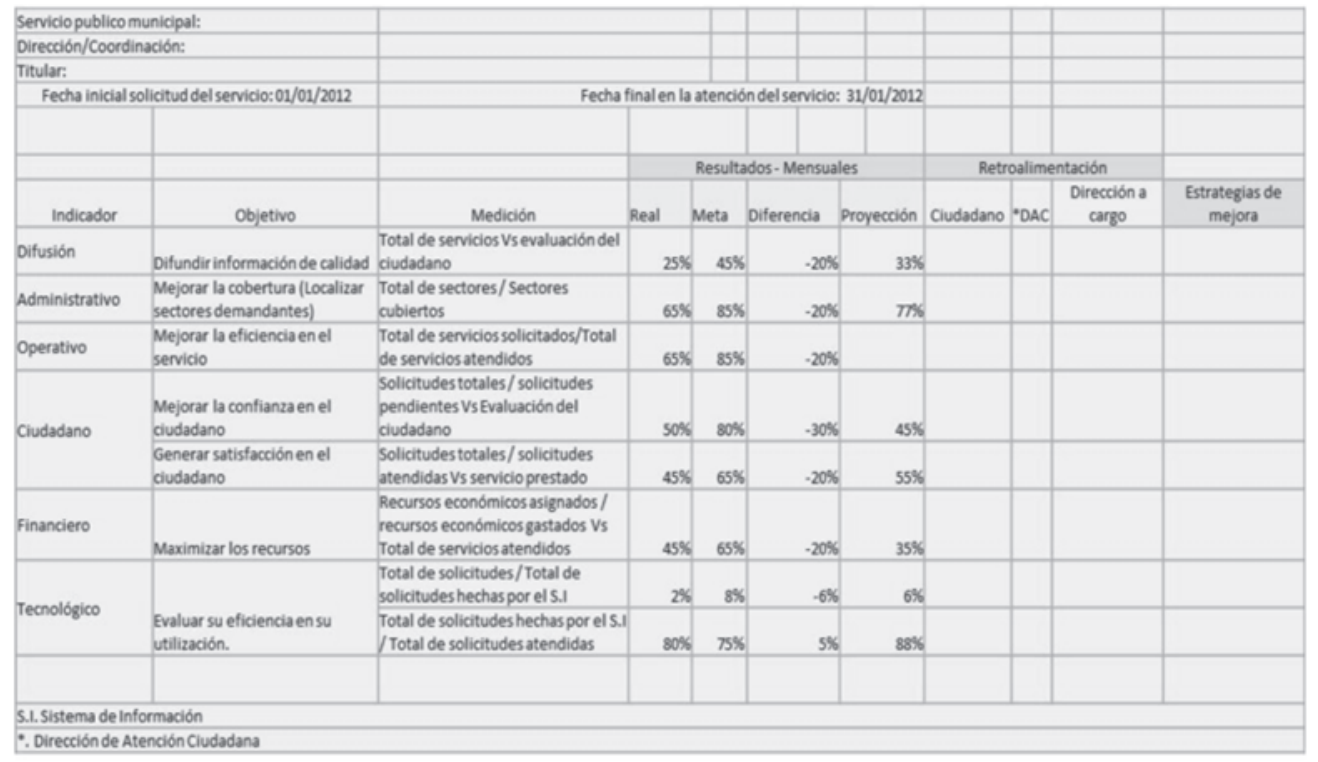

Fuente: elaboración propia (2012). 
A continuación se muestran en la llustración 8, los aspectos que se consideran relevantes para el componente educativo.

Ilustración 8. Aspectos que se consideran relevantes para el componente educativo

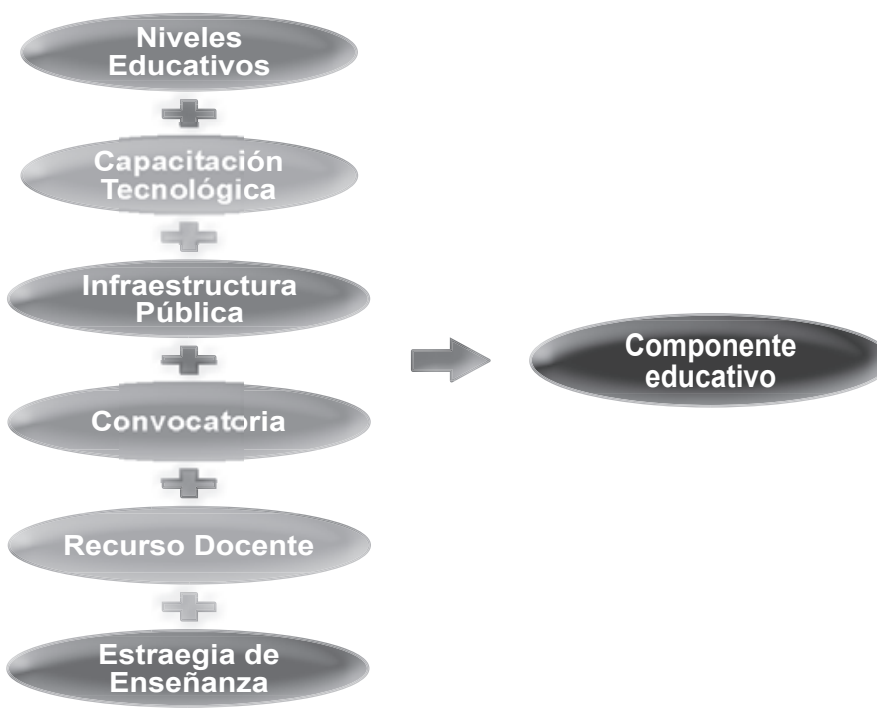

Fuente: elaboración propia (2012).

\section{Componente tecnológico}

Tiene como objetivo realizar un análisis detallado de las necesidades que se requieren para implementar sistemas de información para el control de gestión de servicios públicos municipales, proyectando dicho análisis a corto, mediano y largo plazo. Además de seleccionar las herramientas de desarrollo, los niveles de seguridad que requiera el sistema de información, que forma parte del componente tecnológico, así como los diagramas de casos de uso que ejemplificarán de manera detallada y general los procedimientos y alcances del sistema.

Además se busca la mejor manera de que el componente tecnológico sea fácil de usar y así propiciar su retroali- mentación e interacción entre los actores que intervienen en el proceso del control de gestión de dichos servicios públicos. Se presenta a continuación la llustración 9 del componente tecnológico, y los aspectos que se proponen deberán de contener.

Finalmente, se presenta la ilustración 10, con los cuatro componentes y su interacción, con el propósito de mejorar el control de gestión de los servicios públicos municipales. Como se puede observar en dicha ilustración, todos los componentes están ligados, y dependen para su implementación, que cada uno de ellos se ejecute de manera satisfactoria y de manera paralela, ya que no se recomienda implementar aisladamente cada componente, ocasionando que no se obtengan los resultados esperados. Adicional a ello, es importante señalar que la implementación de los cuatro componentes se propone realizarlo de manera paulatina y paralela incluyendo objetivos a corto, mediano y largo plazo, en que estén incluidos todos los componentes.

Cabe señalar que en México, no existe un modelo similar al propuesto. Los municipios de Ecatepec, Nezahualcóyotl y Coacalco no cuentan con ningún componente, se trabaja de manera aislada y de manera tradicional. En el caso de los municipios de Querétaro y la Delegación Miguel Hidalgo contemplan parte de los componentes pero se incluyen de manera aislada.

\section{Estudio Beneficio / costo}

Los proyectos del sector público, los utilizan y los financian los ciudadanos de cualquier nivel en el gobierno; mientras que los proyectos en el sector privado son propiedad de las corporaciones, asociaciones e individuos. Los productos y servicios de los proyectos del sector privado los utilizan consumidores y clientes de manera individual. Los organismos del estado proporcionan los mecanismos para incrementar el capital y los fondos de operación para dichos proyectos a través de impuestos, pagos de derechos y préstamos (Blank \& Tarquin, 2006, 322 - 348).

Ilustración 9. Aspectos que integran el componente tecnológico

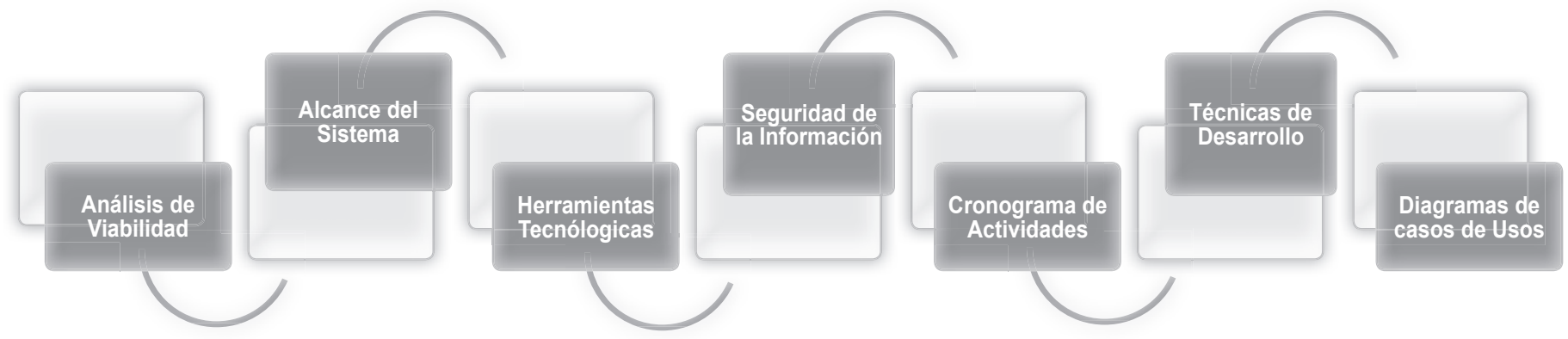

Fuente: elaboración propia (2012). 
Ilustración 10. Modelo para el control de gestión de los servicios públicos municipales.

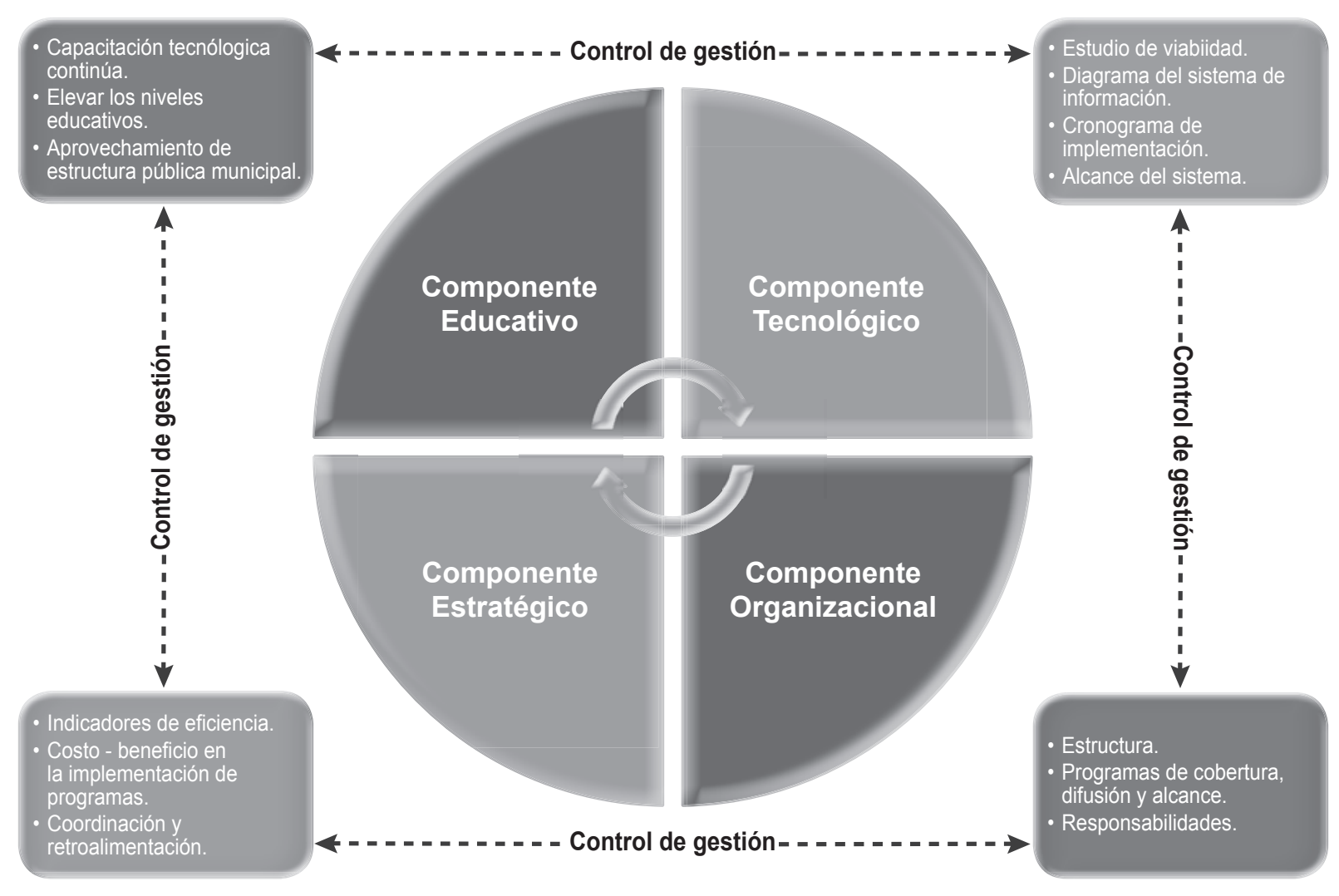

Fuente: Elaboración propia (2012).

La razón beneficio/costo $(B / C)$, se incluye en el presente, con el propósito de mostrar con objetividad el análisis económico de la evaluación del sector público, lo cual reduce el efecto de los intereses políticos y particulares. Según Fabrycky (1997), los proyectos públicos subrayan los beneficios que se le proporcionarán al público por medio de erogaciones propuestas. El método de beneficio-costo calcula la razón de los beneficios de los usuarios al costo del proyecto para el estado. Finalmente, según Baca (2005), los proyectos del sector público, son propiedad del estado y por ende de los ciudadanos, y dichos proyectos producen un beneficio para la misma sociedad, pero también pueden causar contrabeneficios o pérdidas, y adicional a ello los costos del proyecto es el dinero invertido por el estado. El análisis Beneficio/Costo que se propone a continuación, se realizará por cada uno de los componentes propuestos en el modelo, derivado de la información del presupuesto de egresos municipal 2013, según la Dirección de egresos del municipio de Ecatepec de Morelos, Estado de México, El cual haciende a $\$ 3,183,821,370$.

En dichas propuestas se incluirán los diferentes objetivos que persiguen cada uno de los componentes. También se sugiere un último análisis, considerando las cuatro propuestas, a pesar de que la propuesta de que el beneficio / costo del componente estratégico no contemple algún costo, sino por el contrario, éste sirva para ahorrar o distribuir recursos de los demás componentes.

\section{Beneficio / Costo del componente organizacional}

En relación a la creación de una Dirección de Atención Ciudadana de los SPM, pretende lo siguiente:

Objetivo 1. Creación de la Dirección de Atención Ciudadana de SPM.

Objetivo 2. Economía de recursos para el gobierno municipal.

Objetivo 3. Aprovechamiento de recursos para la implementación de programas de difusión, cobertura y alcance.

La propuesta contempla un costo equivalente a $\$ 1.925 .472 .00$ anuales. Ya que solo se requiere contar con 32 recursos, distribuidos de la siguiente manera: 
Tabla 2. Distribución de recursos y montos propuestos para el personal de SPM.

\begin{tabular}{|l|c|c|c|}
\hline \multicolumn{1}{|c|}{ Puesto nuevo } & Puesto según tabulador & Número de RH & Remuneración mensual neta \\
\hline $\begin{array}{l}\text { Dirección de Atención Ciudadana de } \\
\text { Servicios públicos municipales }\end{array}$ & Coordinador & 1 & $\$ 16,456.00$ \\
\hline Departamentos & Subcoordinadores & 3 & $\$ 9,000.00$ \\
\hline Operación AC & Operación & 8 & $\$ 4,500.00$ \\
\hline Operación Coordinación & Operación & 8 & $\$ 4,500.00$ \\
\hline Operación Difusión & Operación & 12 & $\$ 4,500.00$ \\
\hline Total & & 32 & $\$ 1,956,000.00$ \\
\hline
\end{tabular}

Fuente: elaboración propia (2013) con base a la información del tabulador por puesto, obtenida de http://www.ecatepec.gob.mx/transparencia/ operativos.php, consultada el 09/01/2013.

Se presenta a continuación el organigrama de la DACSPM con la distribución de los recursos propuestos.

Organigrama 1. Propuesta de distribución de recursos de la Dirección de Atención Ciudadana de SPM

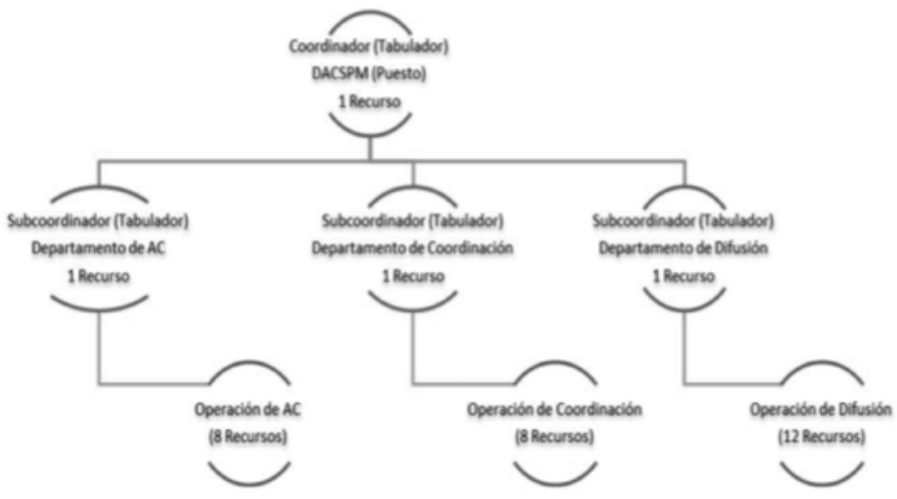

Fuente: Elaboración propia (2013)
Se presentan en la Tabla 3, los siguientes costos asociados a la renta del local, donde se propone ubicar a la dirección propuesta.

Se presentan a continuación en la Tabla 4, los costos asociados a la propuesta de los programas de difusión y cobertura.

\section{Beneficio / Costo del componente estratégico}

El componente estratégico no representa ningún costo, ya que su implementación es más bien cualitativa y está integrada al B/C del componente tecnológico. Sin embargo los resultados de dichos indicadores, pueden representar objetivos del análisis B / C, como son: economizar y distribuir de recursos económicos y materiales, dependiendo del análisis que se realice de dichos resultados. Se recomienda que dichos objetivos sean de manera paulatina, con el propósito de realizar los análisis antes, durante y después de su ejecución

Tabla 3. Concepto y monto de los costos asociados a la renta del local.

\begin{tabular}{|c|c|c|l|l|}
\hline Capítulo & Subcapítulo & Clave & \multicolumn{1}{|c|}{ Concepto } & \multicolumn{1}{|c|}{ Monto } \\
\hline 5000 & 5700 & 5701 & Edificios y locales & $\$ 132,240.00$ \\
\hline 3000 & 3100 & 3106 & Servicio de energía eléctrica & $\$ 14,400.00$ \\
\hline 3000 & 3100 & 3107 & Servicio de agua potable & $\$ 1,500.00$ \\
\hline 3000 & 3100 & 3104 & Servicio telefónico convencional & $\$ 51,600.00$ \\
\hline 5000 & 5300 & 5300 & Vehículos y equipo de transporte & $\$ 10,800.00$ \\
\hline & Total & & & $\$ 210,540.00$ \\
\hline
\end{tabular}

Fuente: elaboración propia (2013), ${ }^{*}$ Catálogo general de claves para estadísticas de las finanzas públicas municipales (2010). catálogo_EFIPEM.pdf, www3. Inegi.org.mx. 
Tabla 4. Detalle de claves y montos de los costos:

\begin{tabular}{|c|c|c|l|l|}
\hline Capítulo & Subcapítulo & Clave & \multicolumn{1}{|c|}{ Concepto } & \multicolumn{1}{c|}{ Monto } \\
\hline 3000 & 3500 & 3506 & Mantenimiento y conservación de vehículos & $\$ 1,455,166.86$ \\
\hline 3000 & 3100 & 3105 & Servicio de radiolocalización & $\$ 48,000$ \\
\hline 2000 & 2100 & 2105 & Materiales y útiles de impresión y reproducción & $\$ 161,280$ \\
\hline \multicolumn{4}{|l|}{ Total } & $\$ 1,664,446.86$ \\
\hline
\end{tabular}

Fuente: Elaboración propia (2013), con base al catálogo general de claves para estadísticas de las finanzas públicas municipales (2010). catálogo_EFIPEM.pdf, www3. Inegi.org.mx. consultado 14/01/2013

\section{Beneficio / Costo del componente educativo}

En relación a este apartado, tendría los siguientes objetivos.

- Objetivo 1. Aprovechar el uso del componente tecnológico, capacitando al personal de la DACSPM

- Objetivo 2. Elevar los niveles educativos.

Se presenta en la Tabla 5 el detalle de los costos asociados a la capacitación tecnológica del modelo propuesto.

\section{Beneficio / Costo del componente tecnológico}

El componente tecnológico, a pesar de que los costos para el desarrollo del sistema de información estarán a cargo de la Dirección General del Sistema Estatal de
Informática del Estado de México, el municipio deberá de tener la infraestructura tecnológica para poder operar dicho sistema. Es por ello que se propone el siguiente análisis Beneficio / Costo. Objetivos que persigue el análisis:

- Objetivo 1. Incorporar satisfactoriamente el componente tecnológico.

- Objetivo 2. Mejorar la eficiencia en el control de gestión de los SPM

Finalmente se realiza a continuación el análisis Beneficio / Costo de los componentes del modelo, anexando el detalle de las cuentas que se incluyeron en cada uno de ellos. Valdría la pena comentar que el ejercicio de este análisis contempla dos momentos.

Tabla 5. Detalle de claves y montos de los costos de la capacitación tecnológica

\begin{tabular}{|c|c|c|l|l|}
\hline Capítulo & Subcapítulo & Clave & Concepto & \multicolumn{1}{|c|}{ Monto } \\
\hline 1000 & 1500 & 1509 & Apoyo a la capacitación de servidores públicos & $\$ 275,600$ \\
\hline 4000 & 4200 & 4211 & Instituciones y programas de educación & $\$ 3,625,000$ \\
\hline 3000 & 3600 & 3690 & Diversos servicios de difusión e información & $\$ 732,120$ \\
\hline Total & \multicolumn{7}{|l|}{} & $\$ 4,632,720$ \\
\hline
\end{tabular}

Fuente: Elaboración propia (2013), con base al catálogo general de claves para estadísticas de las finanzas públicas municipales (2010). catálogo_EFIPEM.pdf, www3. Inegi.org.mx., consultado 19/01/2013.

Tabla 6. Detalle de claves y montos del componente tecnológico

\begin{tabular}{|c|c|c|l|l|}
\hline Capítulo & Subcapítulo & Clave & \multicolumn{1}{|c|}{ Concepto } & \multicolumn{1}{c|}{ Monto } \\
\hline 2000 & 2100 & 2106 & Materiales y útiles para equipos y bienes informáticos & $\$ 166,346.00$ \\
\hline 3000 & 3100 & 3108 & Servicio de telecomunicaciones & $\$ 10,551$ \\
\hline 3000 & 3500 & 3502 & Mantenimiento de bienes informáticos & $\$ 55,800$ \\
\hline 5000 & 5200 & 5206 & Bienes informáticos & $\$ 382,467.36$ \\
\hline 3000 & 3500 & 3530 & Instalación, reparación y mantenimiento de equipos de cómputo y TIC & $\$ 0.0$ \\
\hline \multicolumn{2}{|l|}{ Total } & & $\$ 615,164.36$ \\
\hline
\end{tabular}

Fuente: Elaboración propia (2013), con base al catálogo general de claves para estadísticas de las finanzas públicas municipales (2010). catálogo_ EFIPEM.pdf, www3. Inegi.org.mx., consultado 19/01/2013. 
El análisis del primer año y análisis del segundo, ya que los costos en este último disminuirán, debido a que a partir del segundo se contemplan gastos de renta y no de adquisición.

\section{El primer año de implementación del modelo:}

Beneficio total de cada componente: $\$ 9.351 .095 .41$

- Componente organizacional

D Beneficios totales netos:

- $\$ 3,880,846.82$

- Componente educativo:

$\checkmark$ Beneficios totales netos:

- $\$ 4,839,690.14$

- Componente estratégico:

$>$ Beneficios totales netos:

- 0
- Componente tecnológico

$\checkmark$ Beneficios totales netos:

- $\$ 630,558.45$

Costo total de cada componente: $\$ 9.078 .871 .22$

- Componente organizacional

$>$ Beneficios totales netos:

- $\$ 3,830,986.86$

- Componente educativo:

$>$ Beneficios totales netos:

- $\$ 4,632,720$

- Componente estratégico:

$\rightarrow$ Costos totales netos:

- 0

- Componente tecnológico

$>$ Beneficios totales netos:

- $\$ 615,164.36$

Tabla 7. Detalle de claves y montos de los beneficios del modelo propuesto.

\begin{tabular}{|c|c|c|c|c|}
\hline Capítulo & Subcapítulo & Clave & Concepto & Monto \\
\hline \multicolumn{5}{|c|}{ Componente organizacional } \\
\hline 1000 & 1100 & 1140 & Servicios educativos & $\$ 1,397,886.79$ \\
\hline 1000 & 1100 & 1150 & Servicios de salud & $\$ 865,358.49$ \\
\hline 1000 & 1100 & 1107 & Fomento económico & $\$ 443,773.58$ \\
\hline 1000 & 1100 & 1111 & Desarrollo social & $\$ 754,415.09$ \\
\hline 1000 & 1100 & 1118 & Registros públicos & $\$ 66,566.04$ \\
\hline 3000 & 3600 & 3605 & $1.8 \%$ de Gastos promedio de publicidad y propaganda & $\$ 352,846.83$ \\
\hline \multicolumn{4}{|l|}{ Total } & $\$ 3,880,846.82$ \\
\hline \multicolumn{5}{|c|}{ Componente educativo } \\
\hline 4000 & 4200 & 4211 & $5.6 \%$ de Instituciones y programas de educación & $\$ 3,050,829.23$ \\
\hline 3000 & 3800 & 3081 & $4.85 \%$ Gastos de ceremonia & $\$ 1,788,860.91$ \\
\hline Total & & & & $\$ 4,832,599.97$ \\
\hline \multicolumn{5}{|c|}{ Componente tecnológico } \\
\hline 3000 & 3600 & 3605 & $2.37 \%$ de Gastos promedio de publicidad y propaganda & $\$ 165,976.79$ \\
\hline 3000 & 3800 & 3801 & $0.45 \%$ de los gastos de ceremonia & $\$ 464,581.67$ \\
\hline \multicolumn{4}{|l|}{ Total } & $\$ 627,632.15$ \\
\hline \multicolumn{4}{|c|}{ Total del beneficios del modelo } & $\$ 9,345,270.81$ \\
\hline
\end{tabular}

Fuente: Elaboración propia (2013), con base al catálogo general de claves para estadísticas de las finanzas públicas municipales (2010). catálogo_ EFIPEM.pdf, www3. Inegi.org.mx., consultado 19/01/2013. 
Tabla 8. Detalle de claves y montos de los costos del modelo propuesto en el primer año.

\begin{tabular}{|c|c|c|c|c|}
\hline Capítulo & Subcapítulo & Clave & Concepto & Monto \\
\hline \multicolumn{5}{|c|}{ Componente organizacional } \\
\hline 1000 & 1100 & 1199 & Dependencias diversas & $\$ 1,956,000.00$ \\
\hline 5000 & 5700 & 5701 & Edificios y locales & $\$ 132,240.00$ \\
\hline 3000 & 3100 & 3106 & Servicio de energía eléctrica & $\$ 14,400.00$ \\
\hline 3000 & 3100 & 3107 & Servicio de agua potable & $\$ 1,500.00$ \\
\hline 3000 & 3100 & 3104 & Servicio telefónico convencional & $\$ 51,600.00$ \\
\hline 5000 & 5300 & 5300 & Vehículos y equipo de transporte & $\$ 10,800.00$ \\
\hline 3000 & 3500 & 3506 & Mantenimiento y conservación de vehículos & $\$ 1,455,166.86$ \\
\hline 3000 & 3100 & 3105 & Servicio de radiolocalización & $\$ 48,000$ \\
\hline 2000 & 2100 & 2105 & Materiales y útiles de impresión y reproducción & $\$ 161,280$ \\
\hline \multicolumn{4}{|l|}{ Total } & $\$ 3,830,986.86$ \\
\hline \multicolumn{5}{|c|}{ Componente educativo } \\
\hline 1000 & 1500 & 1509 & Apoyo a la capacitación de servidores públicos & $\$ 275,600$ \\
\hline 4000 & 4200 & 4211 & Instituciones y programas de educación & $\$ 3,625,000$ \\
\hline 3000 & 3600 & 3690 & Diversos servicios de difusión e información & $\$ 732,120$ \\
\hline \multicolumn{4}{|l|}{ Total } & $\$ 4,632,720$ \\
\hline \multicolumn{5}{|c|}{ Componente tecnológico } \\
\hline 2000 & 2100 & 2106 & Materiales y útiles para equipos y bienes informáticos & $\$ 166,346.00$ \\
\hline 3000 & 3100 & 3108 & Servicio de telecomunicaciones & $\$ 10,551$ \\
\hline 3000 & 3500 & 3502 & Mantenimiento de bienes informáticos & $\$ 55,800$ \\
\hline 5000 & 5200 & 5206 & Bienes informáticos & $\$ 382,467.36$ \\
\hline 3000 & 3500 & 3530 & Instalación, reparación y mantenimiento de equipos de cómputo y TIC & $\$ 0.0$ \\
\hline \multicolumn{4}{|l|}{ Total } & $\$ 615,164.36$ \\
\hline
\end{tabular}

Fuente: Elaboración propia (2013), con base al catálogo general de claves para estadísticas de las finanzas públicas municipales (2010). catálogo_ EFIPEM.pdf, www3. Inegi.org.mx., consultado 19/01/2013.

Se presentan en las Tablas 9 y 10, el costo de cada componente propuesto, en el primer y segundo año de implementación.

Tabla 9. Costo por componente en el primer año

\begin{tabular}{|l|c|l|}
\hline \multicolumn{1}{|c|}{ Descripción } & \multicolumn{1}{c|}{ Costo } & Porcentaje \\
\hline Componente organizacional & $\$ 3,830,986.86$ & $42.20 \%$ \\
\hline Componente estratégico & - & $0.00 \%$ \\
\hline Componente educativo & $\$ 4,632,720.00$ & $51.03 \%$ \\
\hline Componente tecnológico & $\$ 615,164.36$ & $6.78 \%$ \\
\hline Total & $\$ 9,078,871.22$ & $100.00 \%$ \\
\hline
\end{tabular}

Fuente: elaboración propia (2013). Como resultado de la investigación de los recursos que se propone integre cada componente.
Tabla 10. Costo por componente en el segundo año

\begin{tabular}{|l|c|l|}
\hline \multicolumn{1}{|c|}{ Descripción } & \multicolumn{1}{c|}{ Costo } & Porcentaje \\
\hline Componente organizacional & $\$ 2,375,820.00$ & $32.81 \%$ \\
\hline Componente estratégico & - & $0.00 \%$ \\
\hline Componente educativo & $\$ 4,632,720.00$ & $63.98 \%$ \\
\hline Componente tecnológico & $\$ 232,697.00$ & $3.21 \%$ \\
\hline Total & $\$ 7,241,237.00$ & $100.00 \%$ \\
\hline
\end{tabular}

Fuente: elaboración propia (2013). Como resultado de la investigación de los recursos que se propone integre cada componente.

Realizando el ejercicio del análisis Beneficio / Costo del primer año, en el municipio de Ecatepec de Morelos, Estado de México, con el presupuesto de egresos 2013, da como resultado lo siguiente: 


$$
\text { B / C }=\frac{\$ 9,351,095.41}{\$ 9,078,871.22}=1.03
$$

Derivado a que el resultado (1.03) es $>=1.0$, el proyecto es económicamente aceptable.

Realizando el ejercicio del análisis Beneficio / Costo del segundo año, en el municipio de Ecatepec de Morelos, Estado de México, con el presupuesto de egresos 2013, da como resultado lo siguiente:

$$
\text { B / C }=\frac{\$ 9,351,095.41}{\$ 7,241,237.00}=1.29
$$

Derivado a que el resultado (1.29) es $>=1.0$, el proyecto es aceptable. Obteniendo una economía de: $\$ 2.109 .858 .41$., respecto al primer año.

\section{BENEFICIOS ESPERADOS}

Los beneficios que se pretenden obtener son principalmente sociales, ya que por un lado el ciudadano no absorbe los costos de los componentes propuestos, debido a que la propuesta se enfoca en utilizar los recursos con que se cuentan actualmente. Se propone que el desarrollo e implementación del sistema quede a cargo de los municipios, estados y de la federación que tienen ya una infraestructura tecnológica para dar soporte a la propuesta tecnológica y educativa.

- Beneficios sociales. Acercamiento de la tecnología, elevar niveles educativos, difundir información de calidad en todo el territorio mediante programas bien estructurados, tomando en cuenta a los ciudadanos como factor primordial para retroalimentar e interactuar entre las autoridades municipales y los cuatro componentes.

- Beneficio económico. El ahorro de transporte, ya que se pretende con la propuesta implementar programas en donde el ciudadano pueda solicitar el servicio público sin trasladarse físicamente a la dirección que ofrece dicho servicio. Ahorro en papelería y en tiempo, ya que el componente tecnológico estará activo las 24 horas del día, desde cualquier lugar incluyendo la infraestructura pública como escuelas, parques con acceso a internet, bibliotecas con infraestructura tecnológica, programas móviles, etc.

- Beneficios Gubernamentales. Mediante los cuatro componentes propuestos se lograrán varios beneficios: mejorar la eficiencia en el control de gestión de los servicios públicos municipales, eliminar trámites redundantes, que absorberá el componente tecnológico y estratégico. Elevar los niveles educativos de la población (ciudadanos y autoridades municipales). Capacitación tecnológica continua, para el aprovechamiento y retroalimentación de la tecnología que se utilice. Eliminar de manera paulatina el uso de papelería. Evaluar los procedimientos, y al personal en todo el proceso de los servicios públicos municipales. Lograr la retroalimentación entre los involucrados. Acercar la tecnología y brindar alternativas para que el ciudadano tenga varias opciones para solicitar un servicio público municipal.

\section{RESULTADOS}

La modernización de los servicios públicos municipales, está ligada directamente al incremento en la demanda de dichos servicios, a la educación del personal administrativo y de la población, al alcance que se tenga de las tecnologías y finalmente a la incorporación de los sistemas de información, sin dejar a un lado la participación del ciudadano, para que el gobierno se involucre y procure dicha participación.

Es menester de ambos, gobierno y ciudadanos, intensificar las acciones para incorporar tecnologías de la información en beneficio de todos, así como brindar la confianza necesaria en el ciudadano y autoridades municipales, para que a través de cualquier medio de solicitud sean atendidas las demandas de servicios públicos municipales. Las perspectivas en el uso de los sistemas de información son prometedoras, siempre y cuando se inicie un proceso de sensibilización - aprendizaje, brindar los medios tecnológicos necesarios, incrementar los niveles educativos en cada región, así como divulgar por parte de las autoridades municipales la existencia de los sistemas de información como vía para la solicitud, atención y seguimiento de cualquier servicio público municipal.

Por este motivo, los cuatro componentes en conjunto forman parte del modelo, que se propone dará las bases para mejorar/hacer eficiente el control de gestión de los servicios públicos municipales en México y con ello lograr la modernización de éstos.

Finalmente, se presenta a continuación en la llustración 11, la propuesta de un sistema de información que forma parte del componente tecnológico, como una alternativa en el control de gestión de los servicios públicos municipales en México, así como la interacción de éste con las entidades internas y externas.

En este diagrama se planean tres bloques de procesos, uno con los indicadores de eficiencia y objetivos de cada área o dirección, otro con la seguridad de la información y cifrado de datos para garantizar los mismos, y finalmente el mecanismo operativo para la solicitud, seguimiento y finalización de los servicios públicos. 
Ilustración 11. Sistema de Información, para el Control de Gestión de los Servicios Públicos Municipales en México

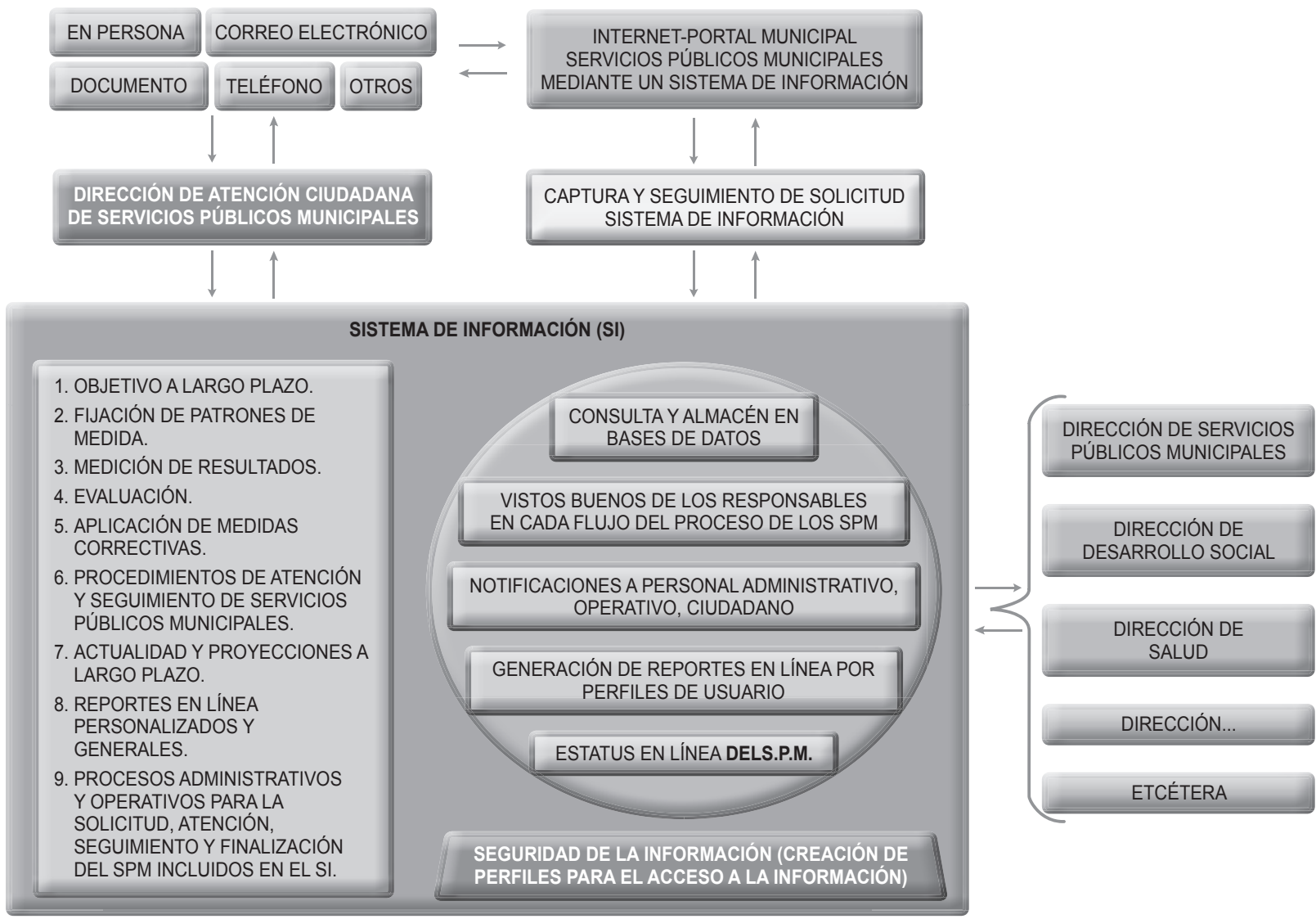

Fuente: Elaboración propia (2012), a partir de: Procesos de control de gestión por el INAP (1986). Cuadro de Mando Integral (Kaplan \& Norton (2002). Manuales de procedimientos de los gobiernos municipales, observados en la investigación de campo. Experiencias de Sistemas de Información para el control de gestión en los municipios investigados.

\section{CONCLUSIONES}

La utilización del modelo, como una herramienta alterna para el control de gestión de los servicios públicos municipales, busca incorporar de manera paralela a cada componente, involucrando a las autoridades y ciudadanos. Creando una cultura de interacción para el ciudadano y autoridades municipales, siendo la retroalimentación parte fundamental de la propuesta, así como dar seguimiento a los cuatro componentes para mejorar cada uno de ellos, o en su defecto proponer modificaciones a los mismos con el objetivo de hacer eficiente el control de gestión de los servicios públicos municipales.

\section{REFERENCIAS}

Andreu, R., \& Valor, J. (1991). Estrategia y Sistemas de Información. Madrid: Mc Graw-Hill - $2^{a}$ Edición.

Baca, C. (2005). Ingeniería Económica. Bogotá Colombia: Fondo Editorial Panamericano.
Gobierno municipal de Ecatepec de Morelos, Estado de México. (2011). Bando Municipal de Ecatepec de Morelos.

Blank P.E, L., \& Tarquin, P.E, A. (2006). Ingeniería Económica. México: MCGraw Hill, Interamericana.

Briones , G. (2002). Metodología de la investigación cuantitativa en las ciencias sociales. Bogotá, Colombia: ARFO.

Castillo de la Peña. (2010). En Metodología para la elaboración del trabajo científico (págs. 228 - 229). México: Instituto Politécnico Nacional.

Dante, C. (2006). Implementacion y debugging (págs. 2034). Chile: Zigzag.

Del Rincón, D., Arnal, A., Latorre, A., \& Sans, A. (1995). Técnicas de investigación en ciencias Sociales (págs. 2526). Madrid: Dykison.

Edwars, C., Ward, J., \& Bythesway, A. (1998). Fundamentos de Sistemas de Información. Madrid: Prentice Hall. 
Fabrycky, W. (1997). Decisiones Económicas, análisis y proyectos. New Jersey, EUA: Editorial Prentice Hall.

Fernández, Narez, \& García. (2008). En Metodología de la investigación en ciencias sociales (págs. 54 - 58). México: Grupo Editorial Patria.

Fernández, Ruíz. (2002). En Servicios públicos municipales (pág. 41). México: INAP - UNAM.

Finanzas públicas municipales - INEGI. (19 de 01 de 2013). catálogo general de claves para estadísticas de las finanzas públicas municipales - EFIPEM. Obtenido de www3. Inegi.org.mx

García Córdoba, F. (2005). La investigación tecnológica. Investigar, Idear e Innovar en Ingenierías y Ciencias Sociales. México: Limusa Noriega.

García, D. (2000). Sistemas de Información en la Empresa. Madrid: Pirámide.

Gaston, C., \& Naser, A. (2012). El desafio hacie el gobierno abierto en la hora de la igualdad. CEPAL, ONU:

CEPAL, ONU.Hernández Sampieri, R., Fernández Collado, C., \& Baptista Lucio, P. (1997). Metodología de la investigación (págs. 28-30), (1ra. Ed.). Colombia: McGraw Hill.
Instituto Nacional de Administración Pública - INAP. (1986). El control de la gestión municipal. México: INAP.Kaplan, R., \& Norton,, D. (2002). Cuadro de mando integral. España: Harvard College.

Laudon, K., \& Laundon, J. (1996). Administración de los Sistemas de Información. México: Prentice Hall.

Montaño Váquez, V. (2008). Modelo de incorporación de TIC's en el proceso de innovación. México: UNAM.

Penso, D. (2011). Cuerpos academicos y grupos de investigación en Analisis organizacional. Mazatlán.

Rodríguez Gómez, G., Gil Flores, J., \& García Jiménez, E. (1996). Metodología de la investigación cualitativa (1). Málaga: ALJIBE.

Sabino, C. (2002). El proceso de Investigación. Caracas: Editorial Panapo.

Serra Rojas, A. (2002). Derecho administrativo. México: Porrúa.

Weissbluth, M. (2008). Gestión y Políticas Públicas. Chile: Universidad de Chile. 